\title{
FPGA-based Implementation of Orthogonal Wavelet Division Multiplexing
}

\author{
Rheza Egha Dwi Rendra Graha ${ }^{1}$, Achmad Ali Muayyadi ${ }^{2}$, Denny Darlis ${ }^{3}$ \\ ${ }^{1}$ Bachelor of Telecom Engineering, ${ }^{2}$ Postgrad of Electrical Engineering, ${ }^{3}$ Diploma of Telecommunication Engineering \\ ${ }^{1,2,3}$ Telkom University \\ Bandung, Indonesia \\ rhezaegha@students.telkomuniversity.ac.id, alimuayyadi@telkomuniversity.ac.id, denny.darlis@tass.telkomuniversity.ac.id
}

\begin{abstract}
The method of multicarrier being developed at this time, namely Orthogonal Frequency Division Multiplexing (OFDM). But in its development, OFDM has several shortcomings. such as the high value of Peak to Average Power Ratio (PAPR) which causes the lower efficiency of power amplifier. An alternative method of multicarrier modulation is Orthogonal Wavelet Division Multiplexing (OWDM). OFDM uses the Inverse Fast Fourier Transform (IFFT) while OWDM uses the Inverse Discrete Wavelet Transform (IDWT). Our previous study showed that the bit error rate of OWDM is relatively equivalent to that of OFDM, while the PAPR value of OWDM is smaller than that of OFDM. This research implemented OWDM using VHDL language to encode each blocks of OWDM on a Field Programmable Gate Array (FPGA). FPGA has many advantages, one of them is the ability to handle the heavy computational load. The results of modeling and simulation were then implemented on the Field Programmable Gate Array (FPGA) board ATLYS Spartan-6 XC6SLX45 CSG324C. The results of this implementation indicated that the design of OWDM prototype algorithm using the Discrete Wavelet Transform (DWT) technique could be implemented on Spartan-6 board ATLYS XC6SLX45 CSG324C. The implementation results showed the resource usage of $13 \%$ on the FPGA board, with a minimum period of $\mathbf{7 5 . 6 6 8} \mathrm{ns}$ and under the working frequency of Spartan-6 FPGA of 13.216 MHz.
\end{abstract}

Keywords-OWDM; PAPR; DWT; FPGA

\section{INTRODUCTION}

With increasing mobile users affecting the decreased bit rates (multi rate) and the limited bandwidth that makes the performance of data transmission decreases. One technique that can handle large amounts of data can be sent quickly and with limited bandwidth is the OFDM multicarrier modulation technique. OFDM is a transmission technique that divides the data-rate stream into a lower data-rate stream, which is then transmitted in multiple sub-carriers and converts the multipath frequency selective fading channel into a flat fading channel [1][2][3]

During the development of multicarrier on OFDM, it turned OFDM there are still shortcomings. One of them limited the ability of IFFT and FFT [2][4]. Because of the large number of sub-carriers produced so that the high PAPR value makes OFDM performance decrease [3]. The multicarrier alternative technique from the previous one is the modulation using the wavelet of OWDM [1]. OWDM is conceptually the same as OFDM, which distinguishes OWDM using wavelet transform so if in OFDM the system is IFFT and FFT while OWDM uses IDWT and DWT. This wavelet supports multicarrier and is applied using a bank filter or $\mathrm{N}$ level [5][6][7].

To prove the existing theory from the studies has been done, it needs to be simulated and implemented. The results from the simulation will be implemented to the VHSIC Hardware Description Language coding language in the Xilinx ISE software. In this research we use Spartan-6 FieldProgrammable Gate Array (FPGA) from Xilinx ${ }^{\mathrm{TM}}$ [8]. With the implementation of OWDM in FPGA hopefully provide real results and appropriate with existing theory.

\section{BASIC THEORY AND CONFIGURATION}

\section{A. Orthogonal Wavelet Division Multiplexing}

Orthogonal Wavelet Division Multiplexing (OWDM) is an alternative multicarrier technique from the previous multicarrier technique that is OFDM where OWDM uses DWT. The intended use of DWT is modulation and its demodulation is captured by wavelets, not by Fourier Transform. OWDM in communication system consists of signal synthesis process in the form of bank filter with multiple input and one output on transmitter. Each input is a sub-symbol of the super-symbol as the output of the modulation scheme. While output is an OWDM signal that represents super-symbol [1].

The synthesis process generates the OWDM signal as a combination of the weighted OWDM pulses. Each weighted OWDM pulse is a representation of the symbol. In the receiver the process of signal analysis using a bank filter, with one input and multiple output.

\section{B. Wavelet Transform}

Wavelet Transform reduces the complexity of the sending and receiving systems for each subcarrier. DWT is used to produce an orthogonal subcarrier. Implementation of discrete wavelet transforms can be done by passing signals into two DWT filters, namely high pass filter (HPF) and low pass filter 
(LPF), where HPF is used to analyze the high frequencies and LPF is used to analyze the low frequencies. The mathematical formula DWT and IDWT can be represented as follows [1][5]:

$$
\begin{aligned}
& \mathrm{c}_{\text {high }}[\mathrm{k}]=\sum_{\mathrm{n}} \mathrm{x}[\mathrm{k}] \cdot \mathrm{g}\left[\begin{array}{cc}
2 \mathrm{k} & \mathrm{n}]
\end{array}\right. \\
& \mathrm{c}_{\text {low }}[\mathrm{k}]=\sum_{\mathrm{n}} \mathrm{x}[\mathrm{k}] \cdot \mathrm{h}[2 \mathrm{k} \quad \mathrm{n}] \\
& x[k]=\sum_{k=-\infty}^{\infty}\left(c_{\text {high }}[k] \cdot g[2 k-n]\right)+\sum_{k=-\infty}^{\infty}\left(c_{\text {low }}[k] \cdot h[2 k-n]\right)
\end{aligned}
$$

where $c_{\text {high }}(\mathrm{k})=$ high frequency signal, $\mathrm{c}_{\mathrm{low}}(\mathrm{k})=$ low frequency signal, $x(k)=$ information signal, $g(k)$ and $h(k)=$ digital filter coefficients.

Equation (1) and (2) are the algorithms of DWT where $c_{\text {high }}[\mathrm{k}]$ convolution occurs in HPF and $\mathrm{c}_{\text {low }}[\mathrm{k}]$ convolution occurs in LPF. Equation (3) is the algorithm of IDWT where $x[\mathrm{k}]$ is the convoluted combination of $c_{\text {high }}[\mathrm{k}]$ and $\mathrm{c}_{\text {low }}[\mathrm{k}]$. The side lobe on the DWT contains very low data and most of the data is carried by the main lobe, so the amount of interference becomes very low [1]. This research uses Haar wavelet type. The simplicity of this Haar wavelet makes the resulting performance excellent and suitable for analytical purposes as performed on this research.

The diagram blocks from DWT and IDWT are show on Fig. 1 and 2. The diagram show how data can be processed in discrete wavelet modulation to produce and reconstruct an OWDM signal. As we can see from the Fig. that information will be mapped into high and low level frequency component based on chosen wavelet. At the end of the diagrams all signals will be added become a superimposed time domain signals.

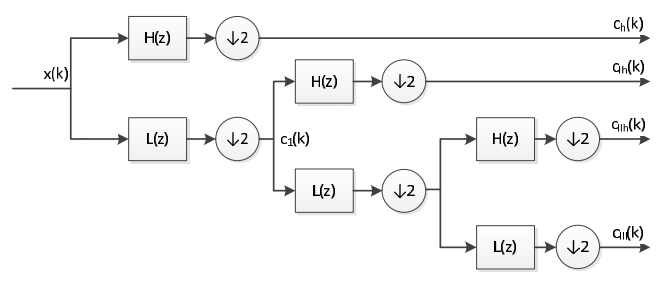

Fig. 1 Block diagram of DWT N-level [6][9]

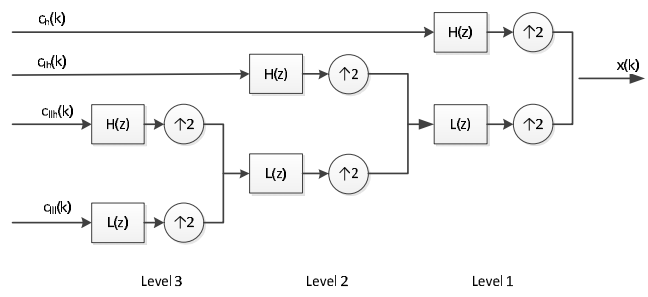

Fig. 2 Block diagram of Inverse DWT N-level [6][9]

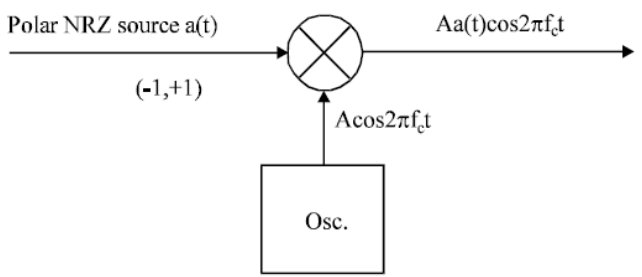

Fig. 3 Block diagram of BPSK modulator [10]

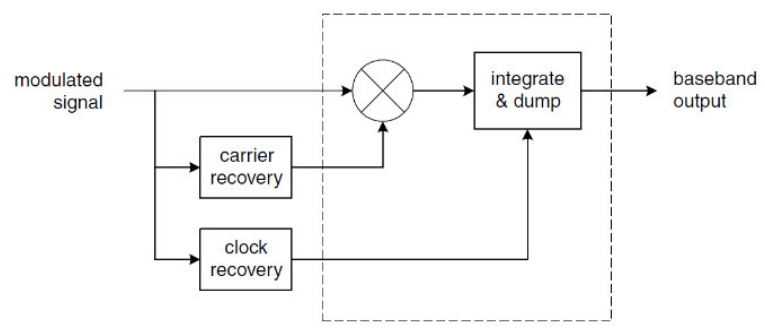

Fig. 4 Block diagram of BPSK demodulator

C. Binary Phase Shift Keying (BPSK)

In BPSK modulation, the carrier frequency is changed between two values denoting the binary state 1 and 0 to the transmission symbol 1 and -1 . In this case the phases of the carrier frequencies differs each other by $\pi$ radians or $180^{\circ}$. In this case the signal on BPSK is also called PRK (Phase Reversal Keying). This signal is used to convey binary digits 0 and 1 respectively. BPSK waveform has the equation as stated on (4).

$$
S(t)=A \cos \left(\omega_{c} t+\theta_{c}\right)
$$

BPSK Modulator has block diagram as shown in Fig. 3.

For demodulation on BPSK is the inverse of BPSK modulation process where the output signal that will enter into BPSK demodulation is changed first to 1 and -1 then it will be translated into 0 and 1 using BPSK constellation diagram. BPSK demodulator has block diagram as shown in Fig. 4.

\section{DESIGN AND SIMULATION}

\section{A. System Diagram}

The modeling of the OWDM system uses DWT, in which the transmitter process is reconstructed with IDWT while the receiver is decomposed with DWT. The number of sub-bands used is 4 . The number of sub-bands depends on the number of levels on DWT filter bank with the formula $(\mathrm{N}+1)$ where $\mathrm{N}$ is the number of levels. Serial to parallel is useful for converting signals from series to parallel and vice versa. In BPSK modulator and demodulator there is a change of analog signal to digital signal and vice versa. Fig. 5 shows a block diagram of OWDM processing[1][6]. 


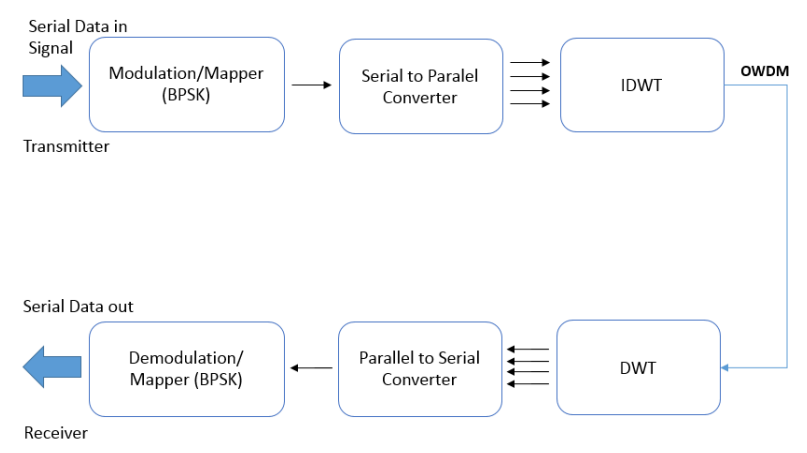

Fig. 5 Block diagram of OWDM transceiver system

\section{B. Simulation Parameters}

Table I is the technical parameters of OWDM using DWT techniques that will be used for the simulation and implementation process.

\section{Flow Chart}

Fig. 6 show the step of OWDM design using DWT techniques that will be used for the simulation and implementation process. From this flowchart it is show that simulation on MATLAB and VHDL Design are used for verify our design.

\section{IMPLEMENTATION AND RESULTS}

\section{A. System Simulation Results on ModelSim $\AA$}

To test the system that has been designed, a test bench that generates a certain row of bits is used. The test bench works on the rising edge clock, meaning the system will be active when the clock changes from ' 0 ' to ' 1 '. To simulate the code, the DWT architecture has been connected with Serial-toParallel as showed on Fig. 7.

Fig. 7 shows that the output data yields the 16 bit data length displayed per-bit with the real value, then the output shows the simulation of the DWT Filter of the bank passed through the Parallel to Serial block. Fig. 8 shows that the output data produces a 1-bit data length on the receiver that has passed the Demodulator / Mapper BPSK block. The whole process from the beginning to the results obtained from OWDM computing resulted in a delay process of $9.422 \mathrm{~ns}$.

Table I Parameter Simulation and Implementation

\begin{tabular}{|c|c|}
\hline Parameter & Unit dan Value \\
\hline Modulation & BPSK \\
Number of sub-band/sub-carrier (N) & 4 \\
Bandwidth & $2 \mathrm{MHz}$ \\
Bit rate (Rb) & $2 \mathrm{Mbps}$ \\
Carrier frequency (Fc) & $2 \mathrm{GHz}$ \\
Delay Spread & $100 \mathrm{~ns}$ \\
Clock Speed & $100 \mathrm{~ns}$ \\
Number of Bit transmitted & 16 \\
Wavelet Filter & Haar \\
\hline
\end{tabular}

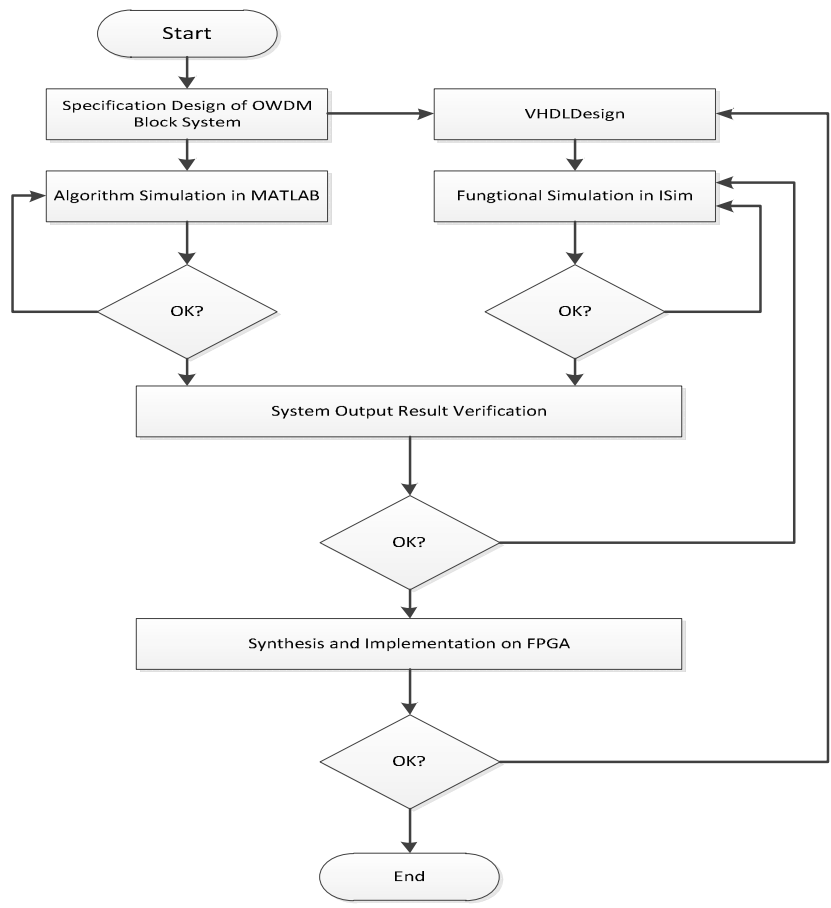

Fig. 6 Flowchart of design and implementation of OWDM system

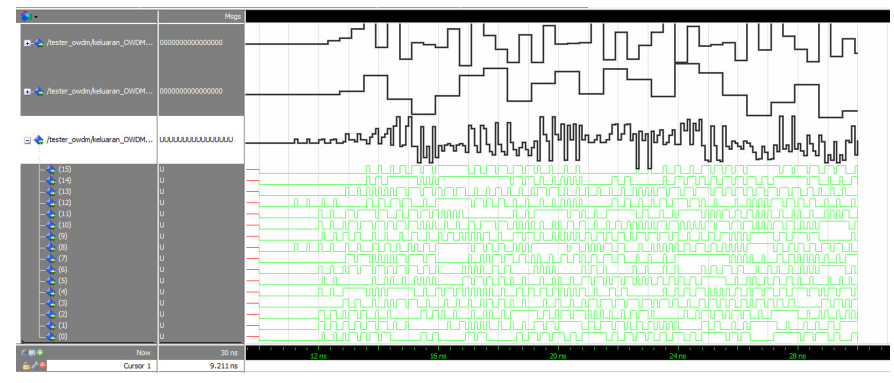

Fig. 7 Simulation of DWT output in ModelSim ${ }^{\circledR}$

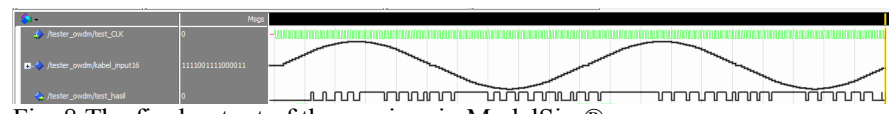

Fig. 8 The final output of the receiver in ModelSim ${ }^{\circledR}$

\section{B. System Utilities}

After designing and tested through simulation, then done synthesis on Xilinx ISE 14.5 software. Table 2 shows the result of the whole system synthesis of OWDM algorithm.

From the results of the synthesis can be obtained some parameters needed in the implementation on the FPGA. From Table 2 it is found that the use of Number of Slices Registers is about $0.133 \%$ of the total resources available. Each slices consists of 2 LUTs and 2 flip-flops. We can see that the Number of Slice LUT's is $52 \%$ and the Number of fully used LUT-FF pairs is $1.188 \%$, it means the design has been designed more as a sequential logic. 


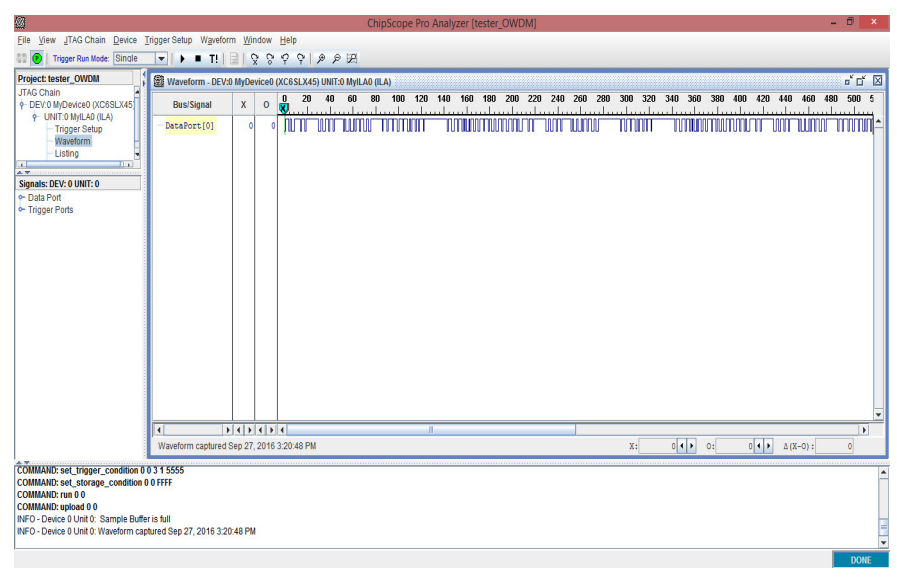

Fig. 9 System output on Xilinx ${ }^{\circledR}$ ChipScope Pro

The synthesis process also gives the result of minimum period and maximum frequency of the system. The minimum period generated is $75.668 \mathrm{~ns}$ and the maximum frequency is 13.216 MHz, so with that frequency the OWDM algorithm prototype using DWT technique can be implemented under the spartan-6 FPGA frequency of $100 \mathrm{MHz}$.

\section{System Simulation Implemented on FPGA}

To find out whether the application program can run and successfully process the data, then used a Xilinx ${ }^{\circledR}$ ChipScope Pro software. When using ChipScope, because the bench test program can be not used in implementation, then used an additional prototype block that serves as a signal generator. Because the signal generator functions to generate rows of bits randomly over a period of time. In prototype signal generator generated signal with 1 bit increments for 10 clock or $100 \mathrm{~ns}$. The process of generating this signal will continue during the clock of the system also continues to run, and the clock condition is the rising edge. Below is an image showing the output of the system on Xilinx ${ }^{\circledR}$ ChipScope Pro.

From Fig. 9 we can see that the ChipScope's output is the same as the simulation output in the ModelSim ${ }^{\circledR}$ software in Fig. 7. So it can be concluded that the OWDM algorithm prototype using DWT Filter Bank which has been designed can be implemented on FPGA ATLYS Spartan-6 XC6SLX45 CSG324C.

Table II Synthesis results of OWDM algorithm design

\begin{tabular}{|l|r|r|r|}
\hline \multicolumn{3}{|c|}{ Device Ulitization } \\
\hline Logic Utilization & \multicolumn{1}{|c|}{ Used } & \multicolumn{1}{|c|}{ Available } & Utilization \\
\hline Number of Slice Registers & 196 & 54576 & $0 \%$ \\
\hline Number of Slice LUTs & 14620 & 27288 & $53 \%$ \\
\hline $\begin{array}{l}\text { Number of fully used LUT-FF } \\
\text { pairs }\end{array}$ & 174 & 14642 & $1 \%$ \\
\hline Number of bonded IOBs & 2 & 218 & $0 \%$ \\
\hline $\begin{array}{l}\text { Number of BUFG, } \\
\text { BUFGCTRL, BUFHCEs }\end{array}$ & 2 & 16 & $12 \%$ \\
\hline Number of DSP48A1s & 12 & 58 & $20 \%$ \\
\hline
\end{tabular}

\section{CONCLUSIONS}

Based on the results of the design and implementation of the OWDM Algorithm that has been done on the Spartan-6 FPGA board, it can be concluded as designing OWDM algorithm using DWT technique can be done up to system functional simulation stage. Designing prototype OWDM algorithm using DWT technique has been successfully implemented on board ATLYS Spartan-6 XC6SLX45 CSG324C. From the simulation result, the system can run the function with total processing time of 9,422 ns where 4,035 ns is logic and $5,387 \mathrm{~ns}$ is route $(42.8 \%$ logic, $57.2 \%$ route). The resulting system has a minimum period of 75,668 ns and working frequency below the working frequency of Spartan-6 FPGA, i.e. 13.216 MHz. With these frequencies still below the availability of resources on the FPGA, the system can be implemented on the board. Design result successfully synthesized and generate OWDM Algorithm resources obtained that the use of Number of Slices Registers about $0.133 \%$ of available resources, $48 \%$ Number of Slice LUTs, $1 \%$ Number of fully used LUT-FF pairs, $0 \%$ Number of bonded IOBs, $12 \%$ Number of BUFG / BUFGCTRL / BUFHCEs and 20\% Number of DSP48A 1s. With the percentage of LUT-FF Slices smaller than LUTs, the prototype is designed more combinational logic than sequential logic.

\section{REFERENCES}

[1] YS Rohmah, A Muayyadi, RP Astuti, "Comparison performance analysis of OWDM and OFDM system on multipath fading Rayleigh channel", Proceedings of Telecommunication Systems Services and Applications (TSSA), 2015.

[2] Denny Darlis, Achmad Ali Muayyadi, Sony Sumaryo., "Perancangan dan Implementasi Prosesor OFDM Baseband untuk Prototipe Modem PLC pada FPGA“, Jurnal Penelitian dan Pengembangan Telekomunikasi, Volume 15, No.2, Desember 2010, Institut Teknologi Telkom.

[3] YS Rohmah, A Muayyadi, RP Astuti, "PAPR comparison Of OWDM and OFDM system", Proceedings of International Conference On Information and Communication Technology (ICoICT), 2014.

[4] Dinda Pramanta, Denny Darlis, Iswahyudi Hidayat, "Simulation of Memory-Based 1024-Point Fast Fourier Transform for Broadband Wireless Access Technology on FPGA“, International Journal of Applied Information Technology, Vol.1 No.2, August 14th, 2017. Telkom University.

[5] A. Graps, "An Introduction to Wavelets, IEEE Computational Science and Eng.", Vol. 2, No. 2 , 1995.

[6] Prof Ali A. A. IEEE, ComSoc, "Discrete Wavelet Transform Based Wireless Digital Communication Systems", Department of Electrical Engineering, Isra University, Amman Jordan, 2010

[7] Burrus, C Sidney, Gopinath, Ramesh A., Guo, Haitao. "Introduction to Wavelet and Wavelet Transform". Prentiice-Hall,Inc., 1998.

[8] Xilinx," 7 Series FPGAs Configurable Logic Block", Xilinx Corp, 2014,

[9] Jatmiko, Wisnu, et.al., "Implementasi berbagai Algoritma Neural Network dan Wavelet pada Field Programmable Gate Array (FPGA)", Fakultas Ilmu Komputer Universitas Indonesia , 2011.

[10] AB. Muhammad, Arfianto Fahmi, Denny Darlis, " Perancangan dan realisasi modulator digital BPSK, QPSK dan 16-QAM pada FPGA", eProceeding of Engineering, Vol.3, No.1, April 2016, pp. 156-163, Universitas Telkom. 